\title{
Safety and efficacy of oral antiplatelet for patients who had acute ischaemic stroke undergoing endovascular therapy
}

Xiaochuan Huo (1) , ${ }^{1}$ Raynald , ${ }^{1}$ Jing Jing, ${ }^{2,3,4}$ Anxin Wang, ${ }^{2,3,4}$ Dapeng Mo, ${ }^{5}$
Feng Gao, ${ }^{5}$ Ning Ma, ${ }^{5}$ Yilong Wang, ${ }^{2,3,4}$ Yongjun Wang, ${ }^{2,3,4}$ Zhongrong Miao (D)

To cite: Huo X, R, Jing J, et al. Safety and efficacy of oral antiplatelet for patients who had acute ischaemic stroke undergoing endovascular therapy. Stroke \& Vascular Neurology 2021;6: e000466. doi:10.1136/svn-2020-000466

- Additional material is published online only. To view, please visit the journal online (http://dx.doi.org/10.1136/svn2020-000466).

$\mathrm{XH}$ and $\mathrm{R}$ - contributed equally.

Received 30 June 2020

Revised 2 August 2020

Accepted 20 September 2020

Published Online First

11 November 2020

Check for updates

(C) Author(s) (or their employer(s)) 2021. Re-use permitted under CC BY-NC. No commercial re-use. See rights and permissions. Published by BMJ.

${ }^{1}$ Neurointervention center, Beijing Tiantan Hospital, Capital Medical University, Beijing

China

${ }^{2}$ Department of Neurology, Beijing Tiantan Hospital, Capital Medical University, Beijing,

China

${ }^{3}$ China National Clinical

Research Center for

Neurological Diseases, Beijing,

China

${ }^{4}$ Center of Stroke, Beijing Institute for Brain Disorders, Beijing, China

${ }^{5}$ Beijing Tiantan Hospital,

Beijing, China

${ }^{6}$ Neurointervention center,

Beijing Tiantan Hospital, Beijing, China

Correspondence to Professor Zhongrong Miao; doctorzhongrongm@126.com

\section{ABSTRACT}

Background and purpose To investigate the safety and efficacy of oral antiplatelet therapy (APT) for patients who had acute ischaemic stroke (AIS), receiving endovascular therapy (EVT).

Methods Patients were divided into non-APT group and APT (single APT or dual APT (DAPT)) group. The safety and efficacy endpoints at 3-month follow-up were symptomatic intracranial haemorrhage (sICH), recanalisation rate, clinical outcome and mortality.

Results Among 915 patients who had AIS, those in APT group $(n=199)$ showed shorter puncture-to-recanalisation time, lower frequency of intravenous thrombolysis and more use of tirofiban compared with those in nonantiplatelet group $(n=716)(p<0.05$ for all). Oral APT was found to be associated with superior clinical outcome compared with non-APT (APT (44.2\%) versus non-APT (41.1\%)), adjusted $\mathrm{OR}=2.605,95 \% \mathrm{Cl} 1.244$ to 5.455 , $\mathrm{p}=0.011)$. DAPT showed superior clinical outcome compared with non-APT (DAPT (56.5\%) versus non-APT (41.1\%), adjusted $\mathrm{OR}=5.405,95 \% \mathrm{Cl} 1.614$ to 18.102 , $\mathrm{p}=0.006$ ) and lower risk of mortality at 3-month followup (DAPT (4.8\%) versus non-DAPT (17.7\%), adjusted $\mathrm{OR}=0.008,95 \% \mathrm{Cl} 0.000$ to $0.441, \mathrm{p}=0.019$ ). There was no significant difference in sICH between the two groups. Conclusions Oral APT prior to undergoing EVT is safe and may accompany with superior clinical outcomes. DAPT may associate with superior clinical outcomes and lower risk of mortality.

\section{INTRODUCTION}

Despite the advantages of antiplatelet therapy (APT) in terms of reducing the risk of stroke and its recurrence, there is still a controversy regarding the potential risk of increased bleeding. ${ }^{1-6}$ To date, a prospective cohort study was conducted to evaluate the safety and efficacy of dual APT (DAPT) prior to intravenous thrombolysis (IVT) for patients who had acute ischaemic stroke (AIS). They reported that DAPT is safe and is not associated with higher rates of bleeding and 3-month mortality. ${ }^{7}$ This result was confirmed by a recent meta-analysis, which demonstrated that the oral antiplatelet is safe and is not associated with higher risk of adverse outcomes in patients who had AIS receiving IVT. ${ }^{8}$
Endovascular therapy (EVT) has been confirmed due to its efficacy to treat AIS with large vessel occlusion in the well-known randomised controlled trials. ${ }^{9-14}$ However, few studies with limited number of patients have evaluated the safety and efficacy of APT for patients who had AIS receiving EVT. ${ }^{15-17}$ Another meta-analysis demonstrated that the randomised controlled trials are warranted to address a question whether the potentially higher risk of symptomatic intracranial haemorrhage (sICH) could be outweighed by improved functional outcome. ${ }^{18}$ Thus, safety and efficacy of APT for patients who had AIS undergoing EVT need to be further assessed.

Hence, the present study aimed to evaluate the safety and efficacy of oral APT for patients who had AIS undergoing EVT.

\section{METHODS}

Study design and patient enrolment

All patients were enrolled from the Acute Ischemic Stroke Cooperation group of Endovascular Treatment (ANGEL) registry, a multicentre nationwide prospective study protocol that recruited 917 patients who had AIS to evaluate the EVT delivery and to improve EVT in clinical practice in Chinese population. The details related to ANGEL study protocol, including design, inclusion/ exclusion criteria, data collection and endovascular procedures, are in accordance with our previous research. ${ }^{19}$

\section{Data collection}

Patient's baseline data, such as age, sex, systolic blood pressure (SBP), the National Institutes of Health Stroke Scale (NIHSS) score, Alberta Stroke Program Early CT Score (ASPECTS), time intervals (onset-todoor (OTD), door-to-puncture, punctureto-recanalisation (PTR), onset-to-puncture and onset-to-recanalisation, were recorded within 24 hours after admission. The assessment of medical records included history of 
atrial fibrillation (confirmed by ECG), diabetes mellitus, previous stroke, hypertension, smoking and drinking. The aetiology of the stroke based on Trial of Org 10172 in Acute Stroke Treatment (TOAST) classification was evaluated by digital subtraction angiography or magnetic resonance angiography or computed tomographic angiography. The data related to the procedural techniques, rescue therapy and administration of heparin and tirofiban during EVT were recorded as well.

All EVT procedures were performed by neurointerventionalist with sufficient experience in neurovascular intervention in mechanical thrombectomy (MT) for AIS. Patients who had AIS undergoing EVT were divided into APT group (single APT (SAPT)/ DAPT) and non-APT group.

\section{Clinical efficacy and safety outcomes}

As a primary safety endpoint, sICH was defined by the European Cooperative Acute Stroke Study III (ECASS-III) trial as evidence of haemorrhage of CT or MR that was felt to be associated with an increase in NIHSS score of $>4$. Functional independence (mRS 0-2) and mortality at 3-month follow-up were taken as primary efficacy endpoints into consideration. However, secondary efficacy endpoint, successful recanalisation was defined as modified Thrombolysis in Cerebral Infarction (mTICI).

\section{Statistical analysis}

The patient's baseline characteristics, including demographic characteristics, vascular risk factors, pathogenesis of stroke according to TOAST classification, clinical and procedural characteristics, stroke categorised as anterior or posterior circulation and OTD, were compared between APT group and non-APT group. The $\chi^{2}$ test was used for making comparison between the two groups, while analysis of variance or Kruskal-Wallis test was employed to compare the baseline characteristics and safety and efficacy outcomes at 3-month among SAPT, DAPT and nonAPT groups. The ORs with $95 \%$ CI of safety and efficacy endpoints (sICH), mTICI grade 2b-3, complete reperfusion (mTICI 3), functional independence (mRS 0-2) and mortality with and without use of APT were evaluated by the logistic regression model. The multivariate models were adjusted for the covariates with $\mathrm{p}<0.20$ in univariate analysis, which included sex, SBP, NIHSS, ASPECTS, atrial fibrillation, hypertension, smoking history, occlusion of the M2 segment of the middle cerebral artery (MCA), IVT, tirofiban and heparin during EVT, general anaesthesia, PTR, MT aspiration, balloon angioplasty and intra-arterial thrombolysis. $\mathrm{P}<0.05$ was considered statistically significant. All statistical analyses were conducted using SPSS software 20.0 software (IBM, Armonk, New York, USA).

\section{RESULTS \\ Patient's baseline characteristics}

Of all 917 patients, 2 patients were excluded from the data analysis due to missing baseline data. Finally, 915 patients who underwent EVT with or without receiving APT were analysed (figure 1). The patient's baseline characteristics are presented in table 1 . The median age was 64 (55-72) years old, $611(66.8 \%)$ patients were male, and 199 (21.7\%) had received APT. In the DAPT group, ASPECT score on admission was relatively higher and hypertension history was more obvious than those in nonAPT and SAPT groups $(\mathrm{p}<0.05$ for all $)$.

The PTR was shorter in the APT group (median (IQR): 70 (44.25 to $110, \mathrm{p}=0.033$ ) and more significant in DAPT group (median (IQR): 65 (30 to 90), $\mathrm{p}=0.001$ ). In the APT group, bridging therapy (IVT followed by EVT) and intra-arterial thrombolysis were less performed compared those in non-APT group $((15.1 \%$ vs $29.5 \%), \mathrm{p}=0.000)$ and $(13.6 \%$ vs $22.6 \%), \mathrm{p}=0.005))$, respectively. On the other hand, the proportions of tirofiban and MT aspiration were found higher in the APT group compared with those in non-APT group (55.8\% vs $27.5 \%, \mathrm{p}=0.000)$ and ( $14.6 \%$ vs $4.3 \%), \mathrm{p}=0.000$ ).

There was no significant difference in vascular risk factors, aetiology of stroke according to TOAST classification, occlusion sites and infarct locations of anterior or

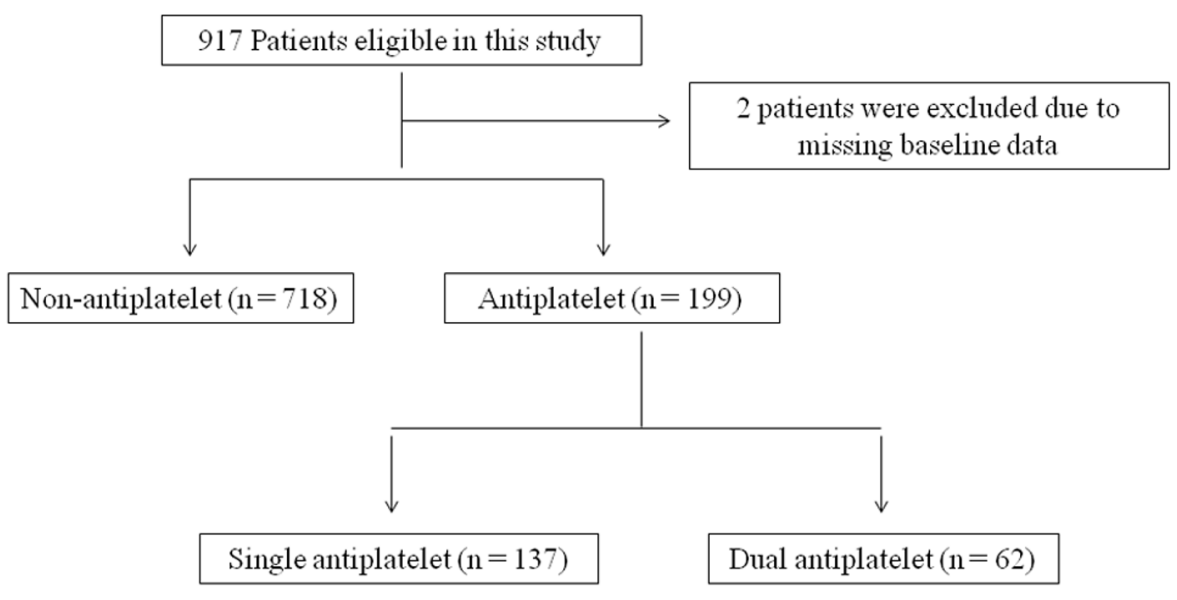

Figure 1 Flowchart showing patient selection. 


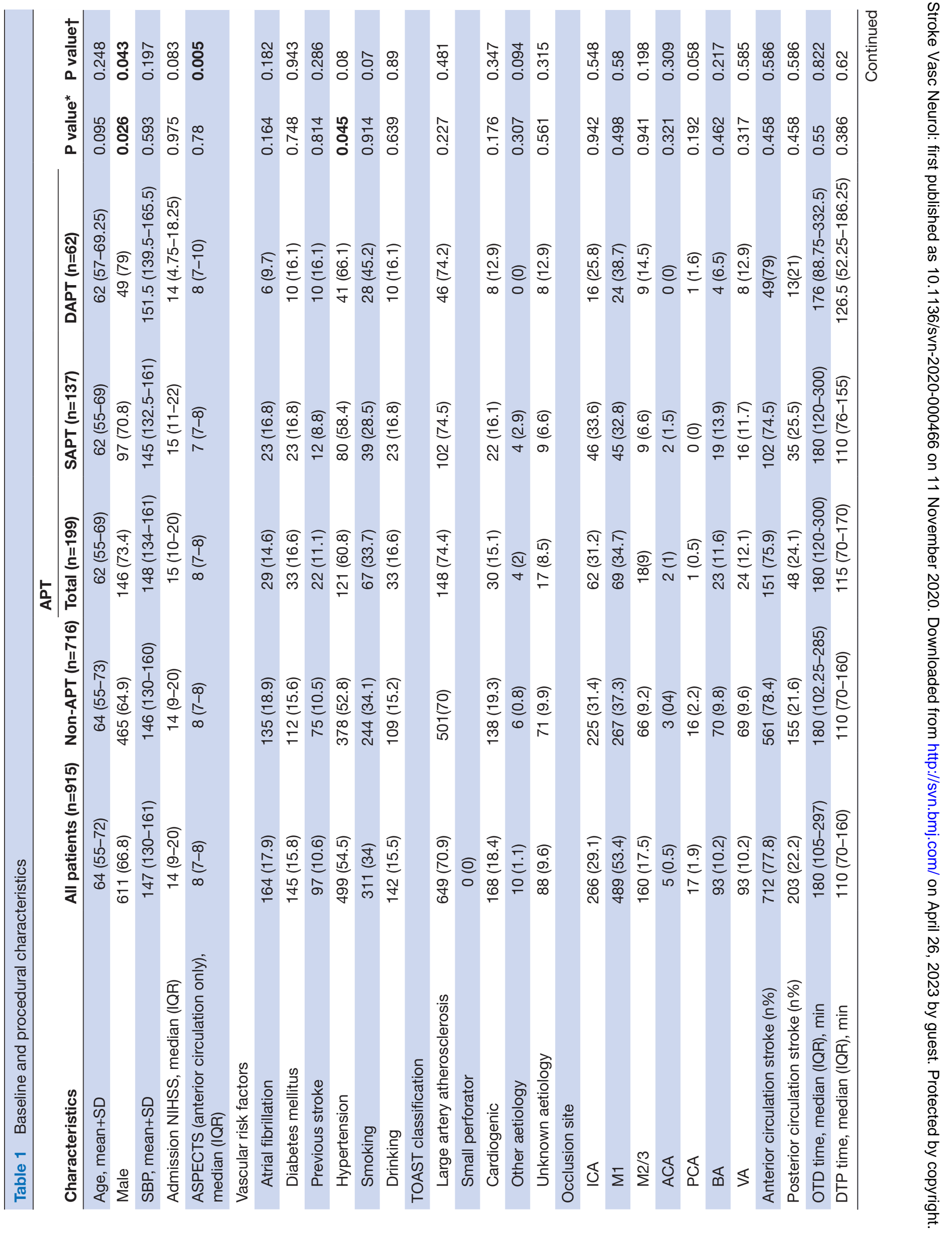




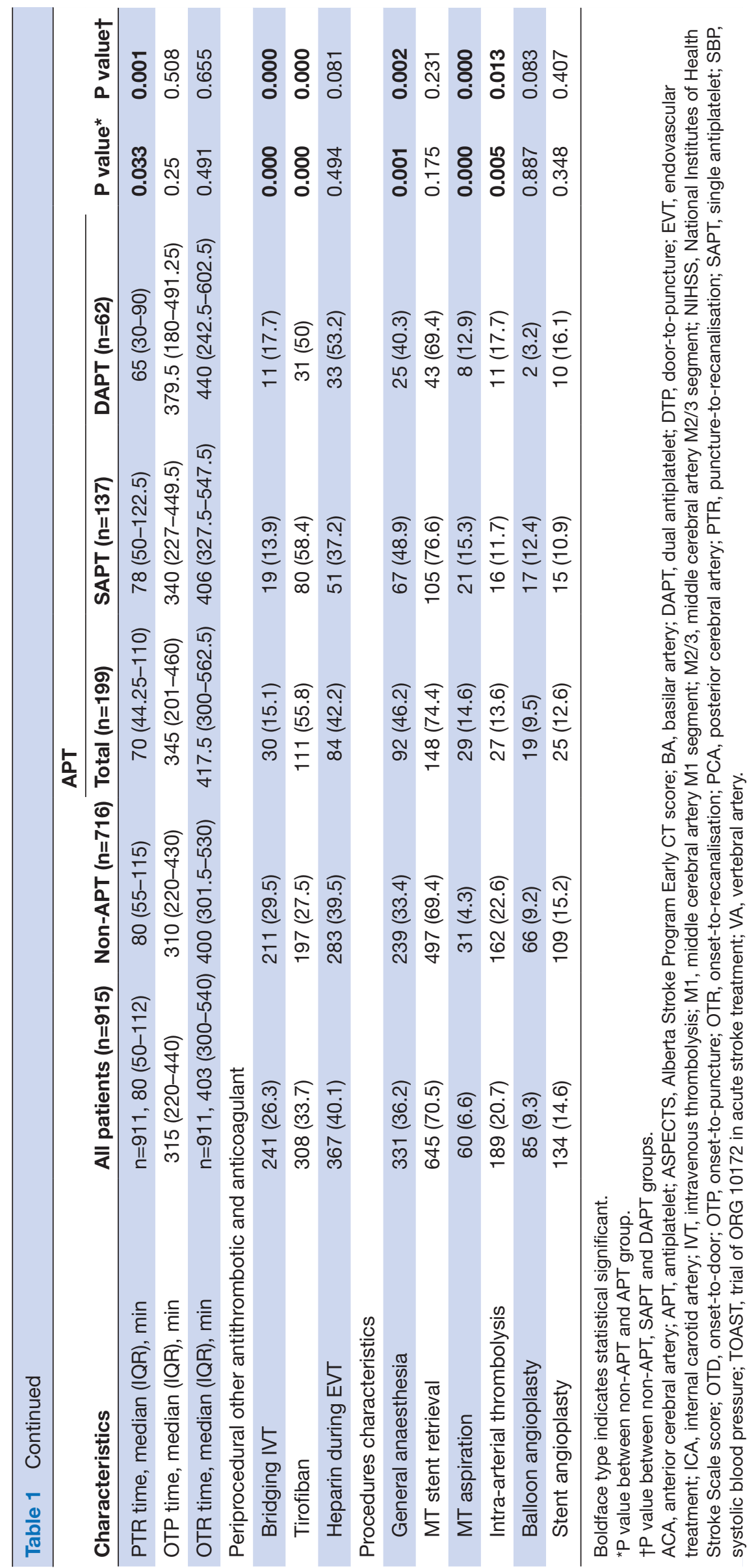


Table 2 Safety and efficacy of pretreatment oral APT in all patients

\begin{tabular}{|c|c|c|c|c|c|c|c|}
\hline \multirow[b]{2}{*}{ Characteristics } & \multirow[b]{2}{*}{$\begin{array}{l}\text { All patients } \\
(\mathrm{n}=915)\end{array}$} & \multirow[b]{2}{*}{$\begin{array}{l}\text { Non-APT } \\
(\mathrm{n}=716)\end{array}$} & \multicolumn{3}{|l|}{ APT } & \multirow[b]{2}{*}{ P value* } & \multirow[b]{2}{*}{$\mathbf{P}$ value } \\
\hline & & & $\begin{array}{l}\text { Total } \\
(n=199)\end{array}$ & $\begin{array}{l}\text { SAPT } \\
(n=137)\end{array}$ & $\begin{array}{l}\text { DAPT } \\
(n=62)\end{array}$ & & \\
\hline $\mathrm{sICH}$ & $51(5.6)$ & $44(6.1)$ & $7(3.5)$ & $3(2.2)$ & $4(6.5)$ & 0.153 & 0.114 \\
\hline \multicolumn{8}{|l|}{ Recanalisation status } \\
\hline $\begin{array}{l}\text { Complete recanalisation } \mathrm{mTICI} \\
3\end{array}$ & $642(70.2)$ & $488(68.2)$ & $154(77.4)$ & $111(81)$ & $43(69.4)$ & 0.012 & 0.01 \\
\hline \multicolumn{8}{|l|}{ Functional outcome at 3 months } \\
\hline Excellent outcome (mRS 0-1) & $382(41.7)$ & $294(41.1)$ & $88(44.2)$ & $53(38.7)$ & $35(56.5)$ & 0.424 & 0.046 \\
\hline
\end{tabular}

${ }^{*} \mathrm{P}$ value between non-APT and APT group.

†P value between non-APT, SAPT and DAPT group.

APT, antiplatelet; DAPT, dual antiplatelet; mRS, modified Rankin score; mTICl, modified treatment in cerebral infarction; SAPT, single antiplatelet; sICH, symptomatic intracranial haemorrhage.

posterior circulation between APT and non-APT groups ( $>0.05$ for all).

\section{Safety and efficacy outcomes}

The safety and efficacy outcomes are shown in tables 2 and 3. Overall, $51(5.6 \%)$ patients developed sICH within 24 hours post-EVT, and no significant difference was noted in sICH incidence between APT group and nonAPT group ( $p>0.05)$. Further analysis demonstrated that there was no correlation between APT with incidence of sICH (adjusted HR, 0.781; 95\% CI 0.103 to 5.944; $p=0.811$ ) even after adjusting for some potential confounders.

As shown in tables 2 and 3, 838 (91.6\%) patients achieved successful recanalisation (mTICI $2 b / 3$ ), while complete recanalisation (mTICI 3) could be attained in $642(70.2 \%)$ patients. However, patients in APT group showed more complete recanalisation compared with those in non-APT group ( $77.4 \%$ vs $68.2 \%, \mathrm{p}=0.012)$, while there was no significant association between APT with successful recanalisation and complete recanalisation even after adjusting for some potential confounders (adjusted HR, 1.410; 95\% CI 0.452 to 4.396 ; $\mathrm{p}=0.554$ and adjusted HR, $1.384 ; 95 \%$ CI 0.628 to 3.053 ; $\mathrm{p}=0.42$, respectively).

At 3-month follow-up, excellent outcome (mRS0-1) and functional independence (mRS0-2) could be achieved in $382(41.7 \%)$ and $473(51.7 \%)$ patients, respectively. However, $160(17.5 \%)$ patients died (mRS 6) at 3-month follow-up (table 2, figure 2). In the current research, superior clinical outcomes and lower risk of mortality were found in patients who received DAPT ( $\mathrm{p}=0.046$ and $\mathrm{p}=0.004$, respectively). In the current research, we noted that DAPT was associated with excellent outcome (mRS0-1) after adjusting for several potential confounders (adjusted OR, 5.405; 95\% CI 1.614 to $18.102 ; \mathrm{p}=0.006$ ). Furthermore, DAPT was significantly correlated with lower risk of mortality (adjusted HR, 0.008; 95\% CI 0.000 to $0.441 ; \mathrm{p}=0.019$ ) even after adjusting for potential factors.

\section{DISCUSSION}

The present study showed that oral APT has a safety outcome over the risk of sICH in patients who had AIS receiving EVT. DAPT indicated a trend of superior clinical outcome and lower risk of mortality.

There are still controversial issues regarding the safety of APT in patients who had AIS receiving recanalisation therapy. On the other hand, some observational studies reported increased risk of sICH following IVT in patients who underwent APT. ${ }^{1-35}$ On the other hand, meta-analyses concluded that APT in patients receiving IVT is not associated with increased risk of sICH even after adjusting for some potential confounders. ${ }^{20}{ }^{21}$ Due to limitations in the number of posthoc analyses, cohort studies and research methodology, a previous study could not confirm the safety of APT following EVT. ${ }^{18}$ In the present study, we found that APT did not increase the risk of sICH. The strength of our finding lies in the larger sample size and adjustment for some potential confounders validating the safety of APT prior to EVT, and previous research studies demonstrated that potential confounders such as, age, vascular risk factors (diabetes mellitus and atrial fibrillation), IVT and intra-arterial thrombolysis are predictors for the increase risk of sICH. ${ }^{22-25}$

There has been limited evidence for the effects of oral APT preceding EVT on the clinical outcomes. Previous studies showed that APT did not improve 
Table 3 Adjusted OR/HR of safety and efficacy outcomes in different treatment groups

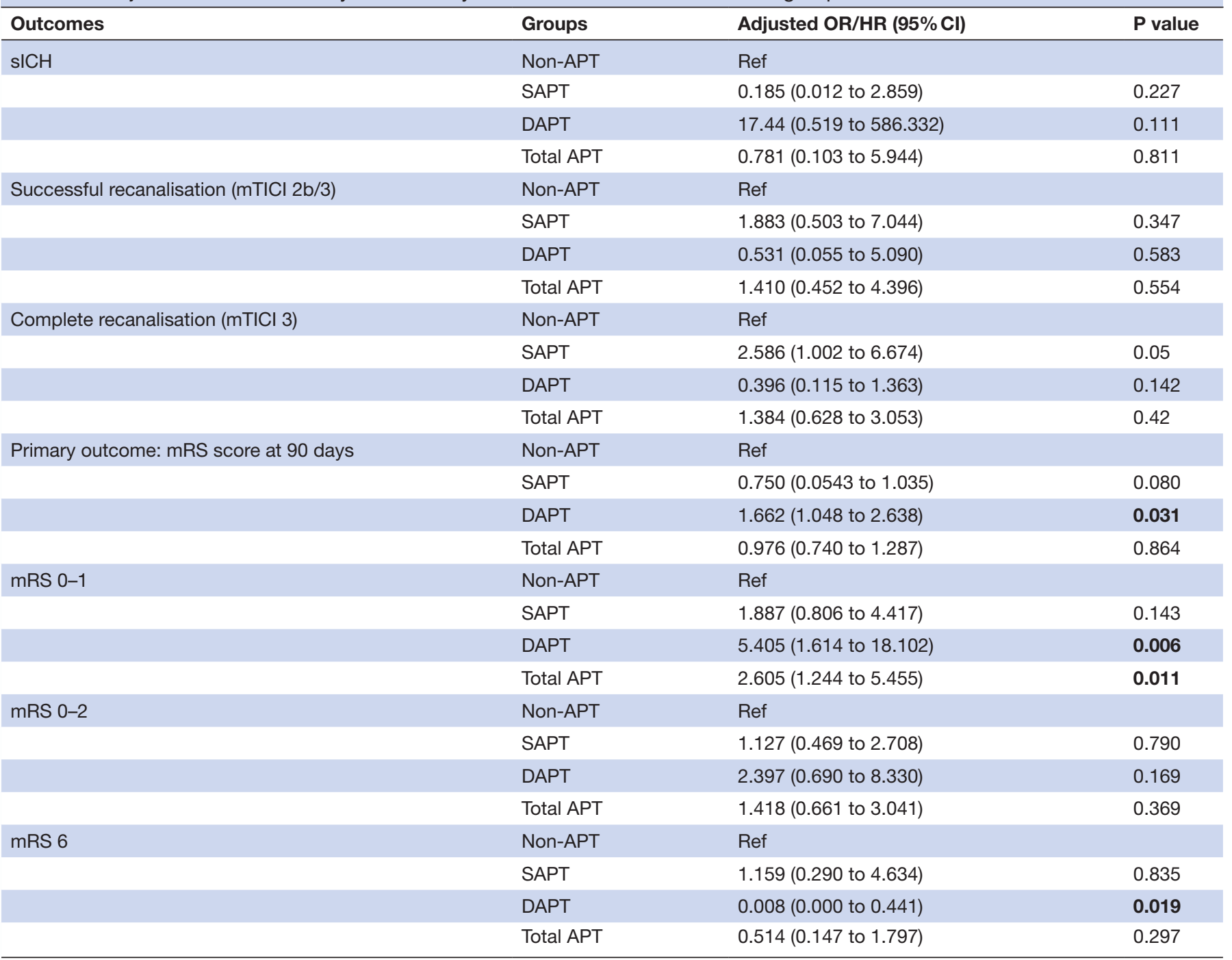

Adjusted for sex, systolic blood pressure, NIHSS, ASPECTS, atrial fibrillation, hypertension, smoking, other aetiology of TOAST classification, MCA M2/3 segment occlusion site, intravenous thrombolysis, tirofiban, heparin use during, puncture to recanalisation, general anaesthesia, mechanical aspiration and intra-arterial thrombolysis and balloon angioplasty.

alCH, asymptomatic intracranial haemorrhage; APT, antiplatelet; ASPECTS, Alberta Stroke Program Early CT Score; DAPT, dual antiplatelet; MCA, middle cerebral artery; mRS, modified Rankin score; mTICl, modified treatment in cerebral infarction; NIHSS, National Institutes of Health Stroke

Scale; SAPT, single antiplatelet; sICH, symptomatic intracranial haemorrhage.

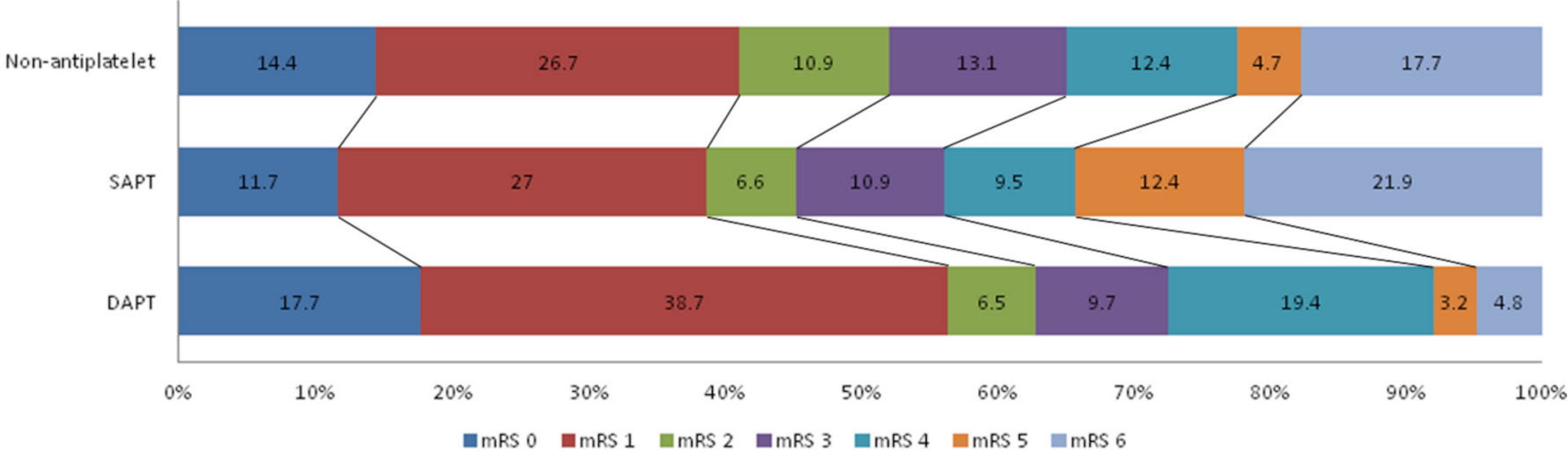

Figure 2 Distribution of mRS scores at 3-month follow-up among non-APT, SAPT and DAPTs. APT, antiplatelet therapy; DAPT, dual APT; mRS, modified Rankin Scale; SAPT, single APT. 
patient's clinical outcomes or reduce the risk mortality at 3-month follow-up although recanalisation could be achieved. ${ }^{15} 17$ On the contrary, the results of the current research showed that DAPT is associated with superior clinical outcomes and lower risk of mortality at 3-month follow-up. This difference might be attributed to the point that the rate of successful recanalisation was higher in the current study compared with that in previous studies ( $91.6 \%$ vs $59 \%-80 \%$, online supplemental table), in which recanalisation is a major predictor of satisfactory outcome following EVT. ${ }^{26} 27$

Nevertheless, special care should be taken during interpretation of our results. Overall, the rate of successful recanalisation was slightly higher in the APT group compared with non-APT group. The positive effects of APT have been well described previously (ie, aspirin may inhibit thrombus formation by interfering the inflammatory and immunological processes, ${ }^{28}{ }^{29}$ and the decrease in tissue factor expression and smoother endothelial generated by antiplatelet may facilitate clot removal and prevent reocclusion after initial recanalisation ${ }^{30}$ ). In addition, the lack of association between APT and recanalisation in the current study might be related to small sample size in DAPT group, requiring further verification in future studies. Nonetheless, we recommended consolidating the use of APT prior to EVT in patients who had AIS. As revealed in the present study, DAPT might shorten the recanalisation time, accompanying with further clinical outcomes. In case of patients with no history of APT, it is recommended to use glycoprotein IIb/IIIa inhibitors, which have more significant dose-dependent blockade effects on platelet aggregation and thrombosis than aspirin or clopidogrel. ${ }^{32}{ }^{33}$ Moreover, the advantages of glycoprotein IIb/IIIa inhibitors may be more significant for Asian population, in which large artery atherosclerosis is the main aetiology of stroke. ${ }^{34}$

Despite the above-mentioned promising result, further randomised controlled trials are required for verification. The strength of the current study lies in the relatively large sample size compared with that in a previous study. Our study has several limitations. First is the uneven distribution between SAPT and DAPT group, which may cause a bias. Second, the EVT and several other rescue therapies were undertaken according to individual experience, which might affect the treatment results. Third, our results cannot be generalised to the global population as the participants enrolled in this study were from China, who have high prevalence of intracranial atherosclerosis. Moreover, the influences of inflammatory factors were not assessed in our research.

\section{CONCLUSION}

In summary, oral APT prior to undergoing EVT is safe and may accompany with clinical outcomes. DAPT may be associated with superior clinical outcomes and lower risk of mortality.
Correction notice This paper has been updated since first published to amend footnotes of Table 1.

Contributors ZM, YW and YW conceived and led the project. XH, R, JJ and AW performed data collection and analysis. DM, FG and NM performed quality control of the data. XH and R cowrote the manuscript with input from all coauthors.

Funding Study funded by the National Key Research and Development Program of China, grant number 2016 YFC1301500.

Competing interests None declared.

Patient consent for publication Not required.

Ethics approval This study was approved by the ethics committee at each participating centre, and informed consent was obtained from all participants prior to starting the study.

Provenance and peer review Not commissioned; externally peer reviewed. Data availability statement Data are available on reasonable request. Requests for access to the data used in this report will be considered by the corresponding author.

Open access This is an open access article distributed in accordance with the Creative Commons Attribution Non Commercial (CC BY-NC 4.0) license, which permits others to distribute, remix, adapt, build upon this work non-commercially, and license their derivative works on different terms, provided the original work is properly cited, appropriate credit is given, any changes made indicated, and the use is non-commercial. See: http://creativecommons.org/licenses/by-nc/4.0/.

\section{ORCID iDs}

Xiaochuan Huo http://orcid.org/0000-0003-1264-5132

Zhongrong Miao http://orcid.org/0000-0003-1970-1221

\section{REFERENCES}

1 Chen S, Lu X, Zhang W, et al. Does prior antiplatelet treatment increase the risk of hemorrhagic transformation and unfavorable outcome on day 90 after intravenous thrombolysis in acute ischemic stroke patients? J Stroke Cerebrovasc Dis 2016;25:1366-70.

2 Enomoto Y, Yoshimura S, Egashira Y, et al. The risk of intracrania hemorrhage in Japanese patients with acute large vessel occlusion; subanalysis of the RESCUE-Japan registry. J Stroke Cerebrovasc Dis 2016;25:1076-80.

3 Dorado L, Millán M, de la Ossa NP, et al. Influence of antiplatelet pretreatment on the risk of intracranial haemorrhage in acute ischaemic stroke after intravenous thrombolysis. Eur J Neurol 2010;17:301-6.

4 Hacke W, Lichy C. Thrombolysis for acute stroke under antiplatelet therapy: safe enough to be beneficial? Nat Clin Pract Neurol 2008;4:474-5.

5 Uyttenboogaart M, Luijckx GJ. Intravenous thrombolysis for patients with ischaemic stroke on antiplatelet therapy: a blessing in disguise? Eur J Neurol 2010;17:177-8.

6 Diedler J, Ahmed N, Sykora M, et al. Safety of intravenous thrombolysis for acute ischemic stroke in patients receiving antiplatelet therapy at stroke onset. Stroke 2010;41:288-94.

7 Tsivgoulis G, Goyal N, Kerro A, et al. Dual antiplatelet therapy pretreatment in IV thrombolysis for acute ischemic stroke. Neurology 2018;91:e1067-76.

8 Malhotra K, Katsanos AH, Goyal N, et al. Safety and efficacy of dual antiplatelet pretreatment in patients with ischemic stroke treated with IV thrombolysis: a systematic review and meta-analysis. Neurology 2020;94:e657-66.

9 Goyal M, Menon BK, van Zwam WH, et al. Endovascular thrombectomy after large-vessel ischaemic stroke: a meta-analysis of individual patient data from five randomised trials. Lancet 2016;387:1723-31.

10 Berkhemer OA, Fransen PSS, Beumer D, et al. A randomized tria of intraarterial treatment for acute ischemic stroke. $N$ Engl J Med 2015;372:11-20.

11 Goyal M, Demchuk AM, Menon BK, et al. Randomized assessment of rapid endovascular treatment of ischemic stroke. $N$ Engl J Med 2015;372:1019-30.

12 Jovin TG, Chamorro A, Cobo E, et al. Thrombectomy within 8 hours after symptom onset in ischemic stroke. $N$ Engl J Med 2015;372:2296-306.

13 Campbell BCV, Mitchell PJ, Kleinig TJ, et al. Endovascular therapy for ischemic stroke with perfusion-imaging selection. N Engl J Med 2015;372:1009-18.

14 Saver JL, Goyal M, Bonafe A, et al. Stent-retriever thrombectomy after intravenous t-PA vs. t-PA alone in stroke. N Engl J Med 2015;372:2285-95. 
15 Pandhi A, Tsivgoulis G, Krishnan R, et al. Antiplatelet pretreatment and outcomes following mechanical thrombectomy for emergent large vessel occlusion strokes. J Neurointerv Surg 2018;10:828-33.

16 Mulder MJ, Berkhemer OA, Fransen PS, et al. Does prior antiplatelet treatment improve functional outcome after intra-arterial treatment for acute ischemic stroke? Int J Stroke 2017;12:368-76.

17 Merlino G, Sponza M, Gigli GL, et al. Prior use of antiplatelet therapy and outcomes after endovascular therapy in acute ischemic stroke due to large vessel occlusion: a single-center experience. J Clin Med 2018;7. doi:10.3390/jcm7120518. [Epub ahead of print: 05 Dec 2018].

18 van de Graaf RA, Chalos V, Del Zoppo GJ, et al. Periprocedural antithrombotic treatment during acute mechanical thrombectomy for ischemic stroke: a systematic review. Front Neurol 2018;9:238.

19 Huo X, Ma N, Mo D, et al. Acute ischaemic stroke cooperation group of endovascular treatment (angel) registry: study protocol for a prospective, multicentre registry in China. Stroke Vasc Neurol 2019;4:57-60.

20 Tsivgoulis G, Katsanos AH, Zand R, et al. Antiplatelet pretreatment and outcomes in intravenous thrombolysis for stroke: a systematic review and meta-analysis. J Neurol 2017;264:1227-35.

21 Luo S, Zhuang M, Zeng W, et al. Intravenous thrombolysis for acute ischemic stroke in patients receiving antiplatelet therapy: a systematic review and meta-analysis of 19 studies. J Am Heart Assoc 2016;5. doi:10.1161/JAHA.116.003242. [Epub ahead of print: 20 May 2016].

22 Lokeskrawee T, Muengtaweepongsa S, Patumanond J, et al. Prediction of symptomatic intracranial hemorrhage after intravenous thrombolysis in acute ischemic stroke: the symptomatic intracranial hemorrhage score. J Stroke Cerebrovasc Dis 2017;26:2622-9.

23 Hao $\mathrm{Y}$, Yang D, Wang $\mathrm{H}$, et al. Predictors for symptomatic intracranial hemorrhage after endovascular treatment of acute ischemic stroke. Stroke 2017;48:1203-9.

24 Gattringer T, Posekany A, Niederkorn K, et al. Predicting early mortality of acute ischemic stroke. Stroke 2019;50:349-56.
25 Mazya M, Egido JA, Ford GA, et al. Predicting the risk of symptomatic intracerebral hemorrhage in ischemic stroke treated with intravenous alteplase: safe implementation of treatments in stroke (SITS) symptomatic intracerebral hemorrhage risk score. Stroke 2012;43:1524-31.

26 Zaidat OO, Yoo AJ, Khatri P, et al. Recommendations on angiographic revascularization grading standards for acute ischemic stroke: a consensus statement. Stroke 2013;44:2650-63.

27 Kleine JF, Wunderlich S, Zimmer C, et al. Time to redefine success? TICI 3 versus $\mathrm{TICl} 2 \mathrm{~B}$ recanalization in middle cerebral artery occlusion treated with thrombectomy. J Neurointerv Surg 2017;9:117-21.

28 Pikija S, Magdic J, Lukic A, et al. Antiplatelet usage impacts clot density in acute anterior circulation ischemic stroke. Int $\mathrm{J} \mathrm{Mol} \mathrm{Sci}$ 2016;17. doi:10.3390/ijms17091382. [Epub ahead of print: 23 Aug 2016].

29 Boeckh-Behrens T, Schubert M, Förschler A, et al. The impact of histological clot composition in embolic stroke. Clin Neuroradiol 2016;26:189-97.

30 Amin AR, Vyas P, Attur M, et al. The mode of action of aspirin-like drugs: effect on inducible nitric oxide synthase. Proc Natl Acad Sci U S A 1995;92:7926-30.

31 Grotta JC, Welch KM, Fagan SC, et al. Clinical deterioration following improvement in the NINDS rt-PA stroke trial. Stroke 2001;32:661-8.

32 Abumiya T, Fitridge R, Mazur C, et al. Integrin alpha(Ilb)beta(3) inhibitor preserves microvascular patency in experimental acute focal cerebral ischemia. Stroke 2000;31:1402-9.

33 Undas A, Brummel-Ziedins KE, Mann KG. Antithrombotic properties of aspirin and resistance to aspirin: beyond strictly antiplatelet actions. Blood 2007;109:2285-92.

34 Yang M, Huo X, Gao F, et al. Low-Dose rescue tirofiban in mechanical thrombectomy for acute cerebral large-artery occlusion. Eur J Neurol 2020;27:1056-61. 